\title{
The impact of speed as well as selected parameters of slot insulation on the distribution of temperature in linear motion converters
}

\author{
JACEK MiKOLAJEWICZ \\ Poznan University of Technology \\ Institute of Electrical Engineering and Electronics \\ Department of Mechatronics and Electrical Machines \\ Piotrowo 3A, 60-965 Poznań, Poland \\ e-mail: jacek.mikolajewicz@put.poznan.pl
}

(Received: 09.05.2016, revised: 13.11.2016)

\begin{abstract}
This paper presents and discusses the mathematical model of thermal phenomena occurring in axis-symmetric electromechanical linear motion converters. On the basis of the developed model, software to analyze the process of the heating up of this type of converters, was created. The effect of the thickness and type of material of the slot insulation, as well as the speed of the runner on the temperature distribution in the analyzed object was examined in-depth. Selected results of simulated calculations have been presented.
\end{abstract}

Key words: thermal fields, Finite Element Method, linear motors, numerical analysis

\section{Introduction}

One of the main criterions limiting the power rating of electromechanical converters, in addition to mechanical strength, is the permissible temperature rise of windings [3]. The improper design of an electromechanical system, in terms of heat, can very quickly lead to its improper functioning, failure and even complete destruction. The ability to analyze devices in terms of heat, during the phase of their design is therefore extremely important. Mathematical models mapping the heating process of thermal phenomena have proven to be very useful.

Many commercial software programs for analyzing the magnetic field in electromagnetic converters are dedicated to the analysis of classic rotating machines. Much less of them however, allow for the analysis of a temperature field. The offer of commercial programs that enable the analysis of a temperature field in systems with linear motion is even poorer. In the case of the classic rotating machines most of the software programs offer preliminarily prepared or ready for calculations machine models (ready patterns). These models frequently allow for a change of geometry, dimensions, material parameters, as well as flexible parame- 
trisation. In the case of transducers with linear motion, in principle, there is no commercial software that contains models which are ready and dedicated to calculations. A model of transducer must be built from scratch, which can also constitute a certain impediment. Therefore you can decidedly more rarely find an analysis of operating and warming-up states for this kind of transducers in the literature. Therefore, the author decided to develop his own, original software for the analysis of thermal phenomena in electromechanical converters of linear motion.

\section{Heat sources in electrical machines}

Heat release in electrical machines, thus the increase of their temperature above the ambient temperature, is associated with the loss of power. In general, total power loss is influenced by several components: these are mainly the power dissipation in the windings (copper) and the magnetic circuit (iron). Furthermore, depending on the type of converter, mechanical (friction in the bearings or friction of the brushes against the commutator or slip rings), ventilation as well as other types of damage (for example in the vat) can occur.

With a constant voltage supply and a constant frequency, the loss of power in the core is virtually the same, irrespective of the load of the machine. The power dissipated in the windings however, varies with regard to the square of the current and is strictly dependent on the load of the device. The heating process of electrical machines and the occurrence of the transfer of heat from a machine to the environment are complex issues. The analysis is made difficult primarily due to the fact that electromechanical converters are not homogenous in terms of heat flow. The presence of high thermal conductivity materials such as copper or iron (Table 1) as well as insulating material of which the coefficient is much smaller, mean that the individual elements of a machine heat up at different rates. Another factor complicating the analysis of this type of system is the mutual exchange of heat between the parts. Because some parts of the machine have an internal heat source and moreover, some elements have better conditions for heat dissipation than others, the interaction of these parts can sometimes be very intense. Varied conditions of heat release into the environment from various surfaces of the machine also lead to uneven temperature distribution in the whole device. This is particularly important for high powered machines.

\section{Mathematical model of thermal phenomena}

Due to the geometry of the tubular linear motor concerned in the article, it is convenient to use a cylindrical coordinate system $(r, z, \varphi)$. The transient temperature distribution can then be described by the relationship $[2,3,5,6]$ :

$$
\nabla \cdot(k(\nabla T(r, z, \varphi, t)))-c_{w} \rho \frac{\partial T(r, z, \varphi, t)}{\partial t}=-Q(r, z, \varphi, t),
$$


where $T$ is the temperature in $\mathrm{K} ; k$ is the coefficient of heat conduction in $\mathrm{W} /(\mathrm{m} \cdot \mathrm{K}) ; c_{w}$ is the specific heat of the material in $\mathrm{J} /(\mathrm{kg} \cdot \mathrm{K}) ; \rho$ is the density of the material in $\mathrm{kg} / \mathrm{m}^{3} ; Q$ is the density of internal heat sources in $\mathrm{W} / \mathrm{m}^{3}$.

In the case of systems with axial symmetry, temperature distribution is the sum of only two spatial coordinates as well as time: $T=f(r, z, t)$. In order to increase the accuracy of the calculations considered, it was taken into account that the relevant object is characterized by high material anisotropy. Taking into account this fact as well as axial symmetry, Equation (1) can be expressed in the form (to simplify the notion: $T=T(r, z, t), Q=Q(r, z, t)$ ):

$$
\frac{\partial}{\partial r}\left(k_{r} \frac{\partial T}{\partial r}\right)+\frac{1}{r} k_{r} \frac{\partial T}{\partial r}+\frac{\partial}{\partial z}\left(k_{z} \frac{\partial T}{\partial z}\right)-c_{w} \rho \frac{\partial T}{\partial t}=-Q
$$

where $k_{r}$ and $k_{z}$ are coefficients of thermal conductivity, in radial and axial directions respectively. However, the calculation assumed the use of isotropic materials. This simplification has been aimed at obtaining a possibly simple calculation model so that the results of simulation calculations obtained from the authorial software can be compared, in an easy and simple manner, with the results from the Comsol Multiphisics commercial software. In the further part of the paper it has been assumed that $k_{r}=k_{z}=k$. Therefore, one $k$ coefficient is in Table 2.

For the conclusive solution of Equation (2), it is necessary to know the limiting conditions, i.e. the initial and boundary conditions. The initial conditions determine the temperature distribution at the initial moment i.e. the assumption of a constant temperature in each point of the object in question, equal to the ambient temperature. While the boundary conditions are the so called demands that this function must meet at edge $S$ of area $\Omega$ at any moment of time.

When dealing with transient heat flow in Equation (2), temperature derivatives with regards to time, appear. For the numerical solution of equations where the derivative of time appears, the "step by step" method was used, in which the temperature value is calculated in successive time points $t_{n}$.

The calculation program adopted the Crank-Nicolson scheme, considered the most accurate. According to this scheme the temperature derivative at the nth time point is calculated as follows:

$$
\left.\frac{\partial T}{\partial t}\right|_{n}=2 \frac{T_{n}-T_{n-1}}{\Delta t}-\left.\frac{\partial T}{\partial t}\right|_{n-1},
$$

where $\Delta t=t_{n}-t_{n-1}$ is the length of the time step.

\section{Simulation results}

The temperature field analysis concerned a three-phase permanent magnet linear synchronous motor (PMLSM). The developed software allows for the changing of any geometric and material parameters of the considered converters with the simultaneous possibility of shaping 
the distribution of the power and heat sources. A structure of an object analysed in the study has been presented in Figure 1, and its geometrical dimensions and material parameters has been summarized in Table 1 and Table 2 .

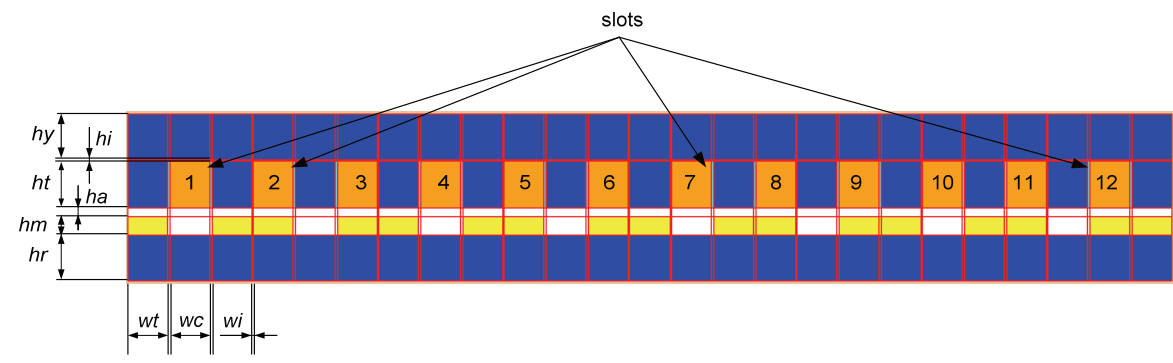

Fig. 1. The structure of the analyzed object

Table 1. Geometrical dimensions

\begin{tabular}{c|c|c}
\hline Description & Symbol & Value $(\mathrm{mm})$ \\
\hline height of the yoke & $h y$ & 10 \\
\hline height of the slot insulation & $h i$ & 0.5 \\
\hline height of the tooth & $h t$ & 10 \\
\hline height of the air gap & $h a$ & 2 \\
\hline height of the magnet & $h m$ & 4 \\
\hline height of the runner & $h r$ & 10 \\
\hline width of the tooth & $w t$ & 10 \\
\hline width of the coil & $w c$ & 10 \\
\hline width of slot insulation & $w i$ & 0.5 \\
\hline & &
\end{tabular}

Table 2. The material parameters of the analyzed object

\begin{tabular}{c|c|c|c}
\hline Material & Density $\left(\mathrm{kg} / \mathrm{m}^{3}\right)$ & $\begin{array}{c}\text { Specific } \\
\text { heat }(\mathrm{J} / \mathrm{kg} \cdot \mathrm{K})\end{array}$ & $\begin{array}{c}\text { Thermal conductivity } \\
\text { coefficient }(\mathrm{W} / \mathrm{m} \cdot \mathrm{K})\end{array}$ \\
\hline Iron & 7974 & 440 & 74 \\
\hline Copper & 8933 & 385 & 400 \\
\hline Air & 1.29 & 1005 & 0.03 \\
\hline Magnet & 7400 & 190 & 18 \\
\hline Epoxy resin & 1200 & 1255 & 0.2 \\
\hline Phenolic resin & 1300 & 1270 & 0.3 \\
\hline
\end{tabular}

To determine the temperature distribution in a linear motor, the Finite Element Method (FEM) was used [1]. The starting point to derive FEM equations, taking into account the combined $2^{\text {nd }}$ and $3^{\text {rd }}$ type boundary conditions, is the dependence on functional energy [5]. For a single finite element it takes the form of: 


$$
I^{(n)}=2 \pi r_{a v} \iint_{\Omega^{(n)}}\left[\frac{k}{2}\left(\left(\frac{\partial T}{\partial r}\right)^{2}+\left(\frac{\partial T}{\partial z}\right)^{2}\right)-Q T+\rho c_{w}\left(\frac{\partial T}{\partial t}\right)\right] \mathrm{d} \Omega^{(n)}+2 \pi r_{a v} \int_{L}\left(q T+\frac{1}{2} h T^{2}-h T T_{\infty}\right) \mathrm{d} L
$$

where $I^{(n)}$ is the functional energy for single finite elements in $\mathrm{W} \cdot \mathrm{K} ; r_{a v}$ is the average value of radial coordinates of single finite element nodes; $q$ in $\mathrm{W} / \mathrm{m}^{2}$ is the heat flow transmitted by a boundary surface; $h$ is the coefficient of heat transfer in $\mathrm{W} /\left(\mathrm{m}^{2} \cdot \mathrm{K}\right) ; T_{\infty}$ is the ambient temperature in $\mathrm{K}$. As a result of object discretization, more than 23,000 finite elements were obtained. By minimizing the functional energy for all finite elements, it is possible to obtain a FEM system of equations in a compact matrix form:

$$
\boldsymbol{S T}+\boldsymbol{C} \frac{\partial}{\partial t} \boldsymbol{T}=\boldsymbol{\Theta}+\boldsymbol{B}
$$

where $\boldsymbol{S}$ is the stiffness matrix; $\boldsymbol{T}$ is a vector of the desired temperature values in the discretized grid nodes; $\boldsymbol{C}$ is a matrix that maps the heat accumulated in the object; $\boldsymbol{\Theta}$ is a vector representing the heat source system; $\boldsymbol{B}$ represents the boundary conditions. To solve the above mentioned system of equations, the Cholesky method was used.

In order to verify the results of the simulation calculations obtained from the authorial software, the author has compared them with the results obtained from the Comsol Multiphysics commercial software. In both cases a thermal steady-state has been analyzed. The results of the calculations have been shown in Figure 2.

a)
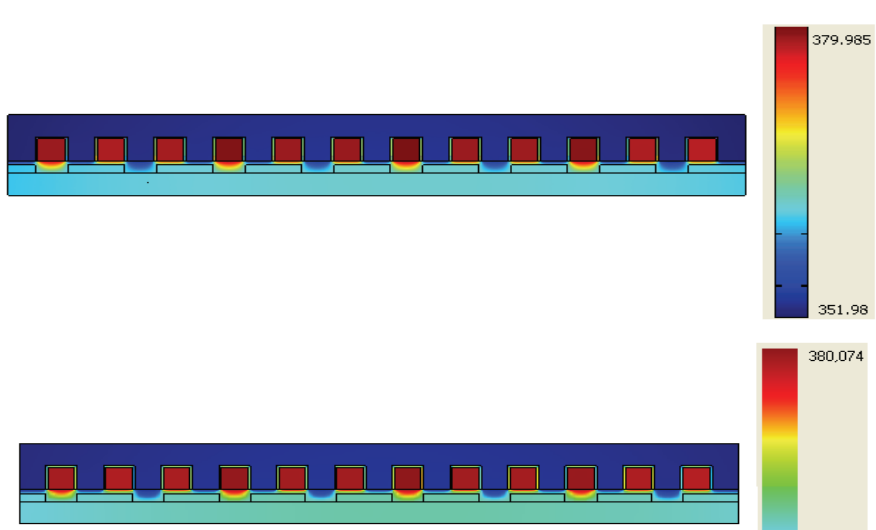

b)

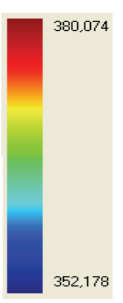

Fig. 2. Calculation results obtained in: a) authorial software; b) Comsol software

While comparing both these figures you can notice that a compliance of the results is very high. The differences between the figures do not exceed $0.1 \%$.

\subsection{Fixed runner}

At the beginning of the simulation tests, the impact of slot insulation heat conductivity on the mean value of the temperature of the windings in slots for several different air gaps was 
examined. It was assumed that the slot insulation is suitably made of epoxy resin, phenolic resin, and "air". The air gap varied in the range of 0.1 to $2 \mathrm{~mm}$. In every case, the same current density of $5 \mathrm{~A} / \mathrm{mm}^{2}$ was forced into the windings. It was assumed that the ambient temperature is $300 \mathrm{~K}$. During the calculations the runner was stationary. The calculation results are shown in Figures 3-5.

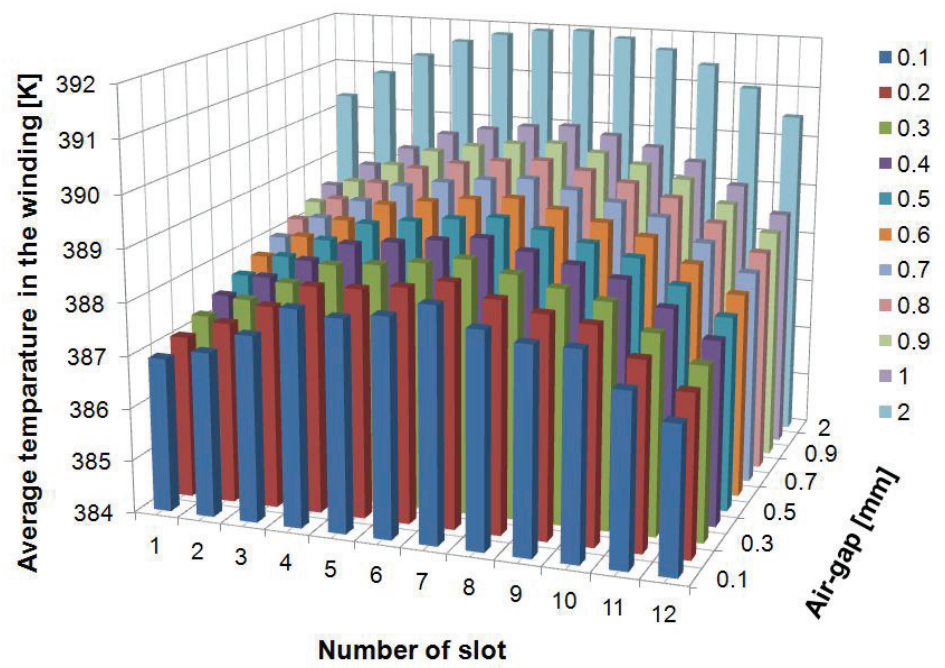

Fig. 3. The average temperature of the windings in slots for several different air gaps with the use of epoxy resin insulation

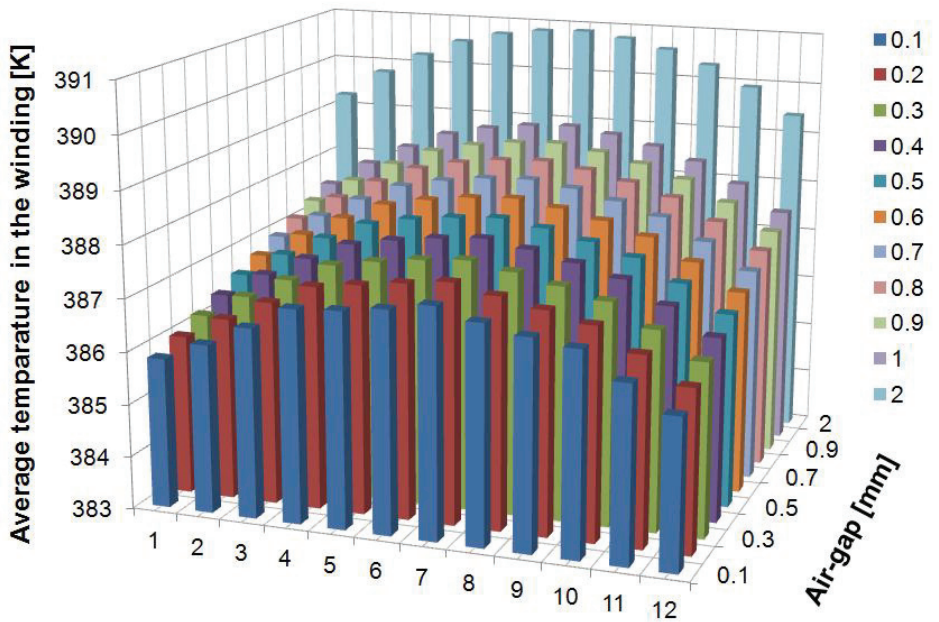

Number of slot

Fig. 4. The average temperature value of windings in slots, for several different air gaps, using phenolic resin insulation 
It is clear to see that the lowest temperature values occur in the case when phenolic resin and a small air gap are applied [2]. With the increase of the air gap, the average temperature in the windings starts to grow. It could be expected that due to the symmetry of the system, the average temperature in slots 6 and 7 should be the same (ignoring numerical rounding errors). The highest temperature however, occurs in the seventh slot. This situation is caused by the arrangement of permanent magnets on the length of the runner.

A different situation applies to the use of "air" insulation. In this case, the impact of the position of the magnets along the length of the runner, on the temperature value is clear. Moreover, the average temperature value is by far the highest, on average, higher by approx. $30 \mathrm{~K}$ compared to the previous two cases.

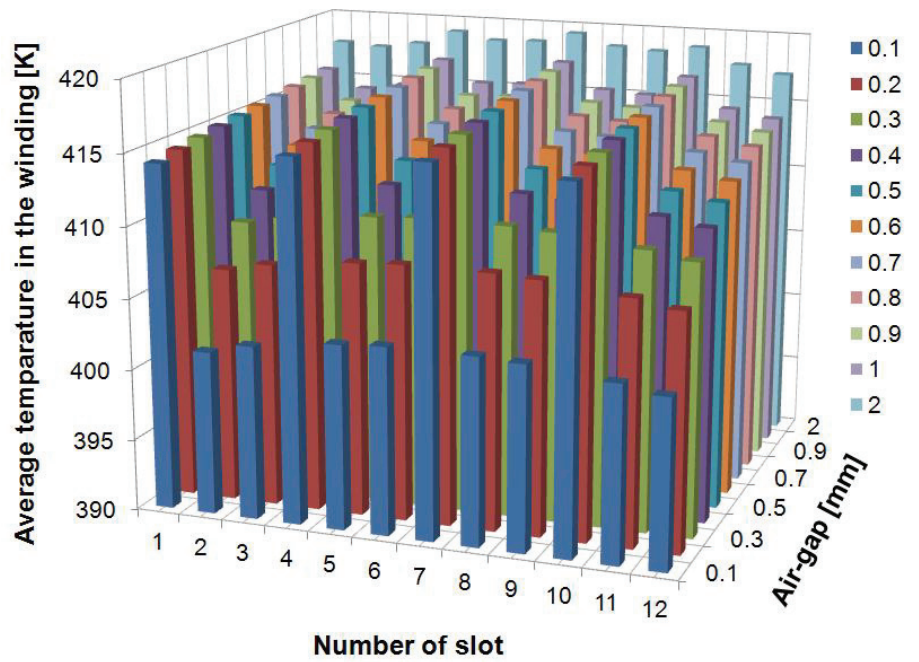

Fig. 5. The average temperature value for windings in slots, for several different air gaps using "air" insulation

The next figure shows the effect of slot insulation thickness on the average temperature value in the slots. Insulation thickness varied in the range of 0.5 to $2 \mathrm{~mm}$. With an increase in slot insulation thickness, there was an increase in the area of the slot. The cross sectional areas of the winding, as well as the current density, were the same as in the previous cases. Calculations were made with a constant air gap thickness of $0.5 \mathrm{~mm}$ and slot insulation made of epoxy resin. It is clear that with an increase in insulation thickness the temperature of the windings decreases. As before, the coils furthest from the center experienced the least heating.

The next figure shows the heating curve of a coil in the seventh slot, relating to the case described above, regarding different slot insulation thicknesses. You can see that the thickness of the slot insulation has virtually no effect on the time constant of the heating process, in the case of the thickest slot insulation $(2 \mathrm{~mm})$, the temperature is set to just a few degrees lower than the worst variant. It would seem that the use of slot insulation, thicker than is required by electrical or mechanical strength is, from the perspective of a rise in temperature, pointless. 


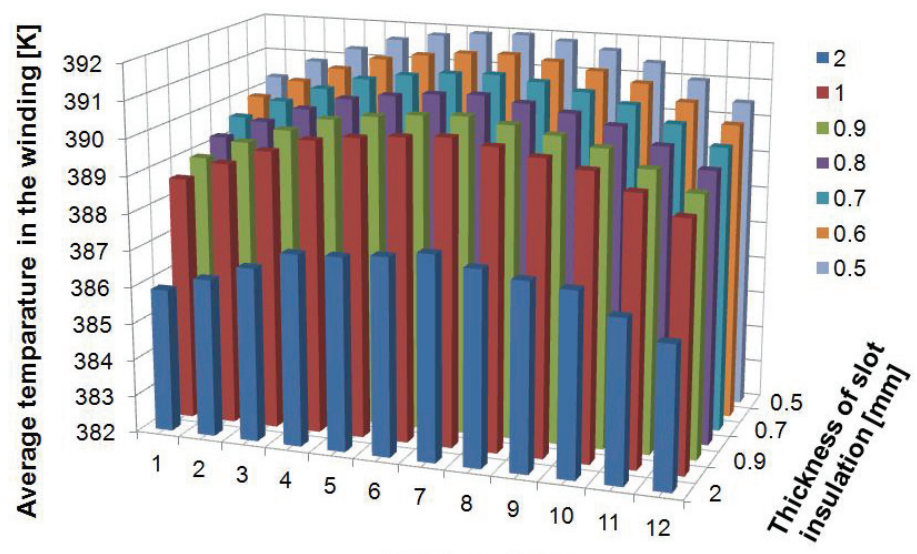

Number of slot

Fig. 6. The average temperature value of windings in slots, for several different slot insulation thicknesses

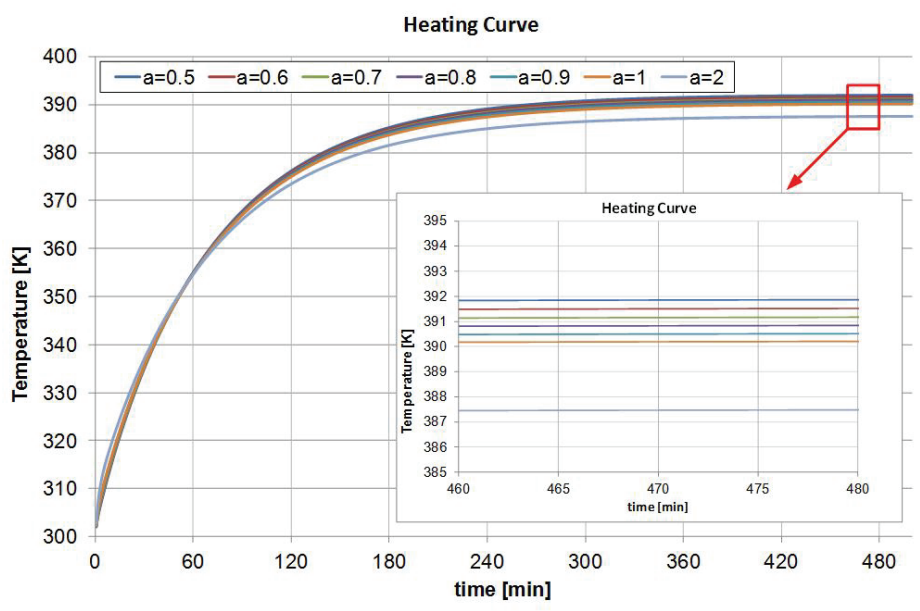

Fig. 7. The heating curves of the seventh slot winding as a function of time for several thicknesses of slot insulation

\subsection{Mobile runner}

The previous considerations related to a case in which the engine runner is stationary. This section examines the impact of linear velocity runner $\mathrm{v}$ on the temperature distribution in a tubular linear motor. In practice, the speed of the mover and thus its influence on the distribution of temperature, are taken into account through the adjustment of the thermal conductivity coefficient in the air gap. It is proposed, that the previously mentioned coefficient, be expressed according to the following relationship [7]:

$$
k_{\mathrm{v}}=k(1+\alpha \sqrt{\mathrm{v}}),
$$


where $k_{\mathrm{v}}$ is the adjusted thermal conductivity coefficient, $\mathrm{v}$ is the linear velocity mover, $\alpha$ is the coefficient characterized by the cooling intensity. The values of $\alpha$ for several different types of cooled surfaces are summarized in Table 3.

\begin{tabular}{l|c}
\multicolumn{1}{c}{ Table 3. The values of $\alpha$ for several different types of cooled surfaces } \\
\hline \multicolumn{1}{c|}{ Type of surface } & $\alpha$ \\
\hline The area thoroughly, evenly and equally washed by the cooling air & 1.3 \\
\hline Frontal connection of windings & 1.0 \\
\hline Cylindrical surface of the rotors & 0.8 \\
\hline Coil surface on the poles & 0.8 \\
\hline Commutator surface & 0.6 \\
\hline The hull surface of traction motors & 0.6 \\
\hline
\end{tabular}

The effect of the speed of the mover on the distribution of temperature in a linear motor is shown below. The calculations were performed for speeds equal to: $0,1,2,4,8$ as well as $10 \mathrm{~m} / \mathrm{s}$, respectively. The cooling intensity coefficient was assumed to be $\alpha=0.8$. For all cases, a common temperature scale was adopted. The minimum value of temperature $T_{\min }$ was $286 \mathrm{~K}$ (dark blue colour); the maximum temperature value $T_{\max }$ was equal to $392 \mathrm{~K}$ (dark red colour). It is clear that with an increase in speed, the temperature of the furthest coils decreases, as does the temperature of the inductor. The temperature of the runner however, increases. This may be relevant for certain types of temperature sensitive magnets. The temperature drop in the slots, mainly six and seven, is very small and doesn't really depend on speed.
a) $\mathrm{v}=0 \mathrm{~m} / \mathrm{s}$
b) $\mathrm{v}=1 \mathrm{~m} / \mathrm{s}$

\section{Dengengenger}

c) $\mathrm{v}=2 \mathrm{~m} / \mathrm{s}$

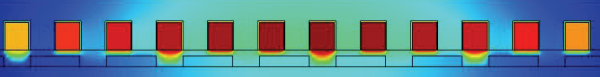

e) $\mathrm{v}=8 \mathrm{~m} / \mathrm{s}$ d) $\mathrm{v}=4 \mathrm{~m} / \mathrm{s}$

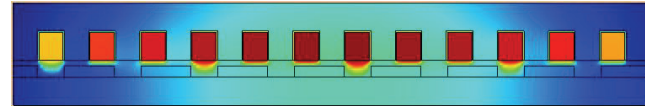

f) $\mathrm{v}=10 \mathrm{~m} / \mathrm{s}$

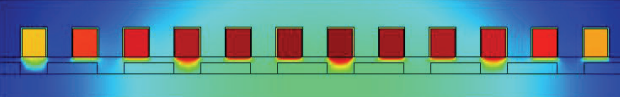

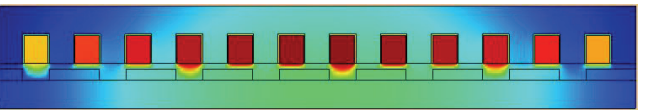

Fig. 8. Effect of the speed of the runner on the temperature distribution

\section{Conclusions}

The developed, original, computer program, enables in-depth analysis of the heating process of electromechanical converters based on linear movement. It allows for the changing of 
any geometrical and material parameters of the object as well as changes in the distribution of the heat sources. It may therefore, be an extremely useful simulation tool already at the design stage of this type of converters. The program, due to the versatility of the developed and implemented procedures and functions, as well as direct access to the program code, can be easily updated and expanded, with for example, electromagnetic or mechanical calculations.

\section{References}

[1] Barański M., FE analysis of current displacement phenomena in a squirrel cage motor working at cryogenic temperature, Archives of Electrical Engineering, vol. 63, no. 2, pp. 139-147 (2014).

[2] Nowak L., Mikołajewicz J., Pietrowski W., Field-circuit model of the thermal phenomena in axialsymmetry electromagnetic devices, Electrical Review (in Polish), ISSN 0033-2097, R. 85 NR 6/2009, pp. 63-66 (2009).

[3] Tomczuk B.Z., Koteras D., Waindok A., Electromagnetic and Temperature 3-D Fields for the Modular Transformers Heating Under High-Frequency Operation, IEEE Transactions on Magnetics, vol. 50, no. 2, February (2014).

[4] Mahdavi S., Herold T., Hameyer K., Thermal Modeling as a Tool to Determine The Overload Capability of Electrical Machines, International Conference on Electrical Machines and Systems, Busan, Korea, pp. 454-458, 26-29 October (2013).

[5] Vong P. K., Rodger D., Coupled Electromagnetic-Thermal Modeling of Electrical Machines, IEEE Transactions on Magnetics, vol. 39, no. 3, pp. 1614-1617, May (2003).

[6] Wiśniewski S., Wiśniewski T.S., Heat transport, Wydawnictwo WNT (in Polish), Warszawa (2012).

[7] Pełczewski W., Heat issues in electrical machines, Wydawnictwo PWT (in Polish), Warszawa (1956). 